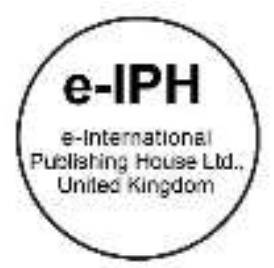

\title{
Correlation Analysis on the Effects of Wearing Compression Socks and Smooth Socks on Running Kinematics among Runners
}

\author{
Muhammad Hanis Mohd Jefry ${ }^{1}$, Hosni Hasan², \\ Raja Mohammed Firhad Raja Azidin ${ }^{3}$ Mohd Azim Nural Azhan ${ }^{4}$
}

${ }^{1}$ Faculty of Sports Science and Recreation, Universiti Teknologi MARA, UiTM, 40450 Shah Alam, Selangor, Malaysia 2Faculty of Sports Science and Recreation, Universiti Teknologi MARA, Perlis Branch, Arau Campus, 02600 Arau, Perlis, Malaysia

hanizjefry@gmail.com, hosnihasan@uitm.edu.my, firhad@uitm.edu.my, azimazhan@uitm.edu.my

Tel of $1^{\text {st }}$ Author: $+6091-3335261$

\begin{abstract}
This study was conducted to determine running kinematics while using compression socks (CS) and smooth socks (SS) among 16 recreational runners. They were required to complete a maximal treadmill test with two different running sock conditions (smooth and compression). All kinematic parameters (ground contact time, heel strike, stride length and swing time) were reported in an average of the four stages of Bruce protocol. Results showed more significant correlations $(p<0.05)$ among the kinematic variables in the compression socks condition as compared to the smooth socks. In conclusion, wearing compression socks improves movement kinematics while running may be due to the enriched somatosensory information received by the foot.

Keywords: Running; Compression socks; Movement kinematics; Somatosensory feedback

eISSN: 2398-4287@ 2021. The Authors. Published for AMER ABRA cE-Bs by e-International Publishing House, Ltd., UK. This is an open access article under the CC BYNC-ND license (http://creativecommons.org/licenses/by-nc-nd/4.0). Peer-review under responsibility of AMER (Association of Malaysian Environment-Behaviour Researchers), ABRA (Association of Behavioural Researchers on Asians/Africans/Arabians) and cE-Bs (Centre for Environment-Behaviour Studies), Faculty of Architecture, Planning \& Surveying, Universiti Teknologi MARA, Malaysia.

DOI: https://doi.org/10.21834/ebpj.v6iSl4.2915
\end{abstract}

\subsection{Introduction}

Compression socks (CS) is one of the popular garments among athletes and is widely used by runners. It is a type of sports garments that were designed to assist athletes in enhancing their sports performance. The use of compression socks can enhance the somatosensory feedback and thus provide better running efficiency among runners (Azhan, Hasan, Azidin, Misnon \& Ismail, 2020). It was made from an elastic material that consists of spandex, nylon, cotton, and natural rubber (Hasan, Davids, Chow, \& Kerr, 2016). The material was snug-fitting and stretchy which will gently squeeze the leg. The socks were tighter at the ankle level and would gradually loosen up as it got closer to the upper part of the leg. Compression socks are worn around the calves which will produce graduated pressure to the calves compared to conventional socks (Areces et al., 2015). As for the sizes, it came in two different lengths which are the knee and ankle length, depending on the user's preference and comfort. The nature of compression socks itself differs from the conventional socks where it compresses the leg more due to its material.

Athletes use compression socks to get similar physiological results as in the medical settings within the context of high sports performance. Some athletes usually use it to enhance their physiological demands and believe that it will improve their performance while others use the socks to prevent further injury. The pressure gradient hypothetically reduces the available space for swelling, aids in the removal of metabolites, and provides mechanical support for the working muscles (Kraemer, French, \& Spiering, 2004). By improving the blood flow, it will affect other demands in sports performance such as endurance and also efficient muscle firing during

eISSN: 2398-4287@ 2021. The Authors. Published for AMER ABRA cE-Bs by e-International Publishing House, Ltd., UK. This is an open access article under the CC BYNC-ND license (http://creativecommons.org/licenses/by-nc-nd/4.0/). Peer-review under responsibility of AMER (Association of Malaysian Environment-Behaviour Researchers), ABRA (Association of Behavioural Researchers on Asians/Africans/Arabians) and cE-Bs (Centre for Environment-Behaviour Studies), Faculty of Architecture, Planning \& Surveying, Universiti Teknologi MARA, Malaysia.

DOI: https://doi.org/10.21834/ebpj.v6iSI4.2915 
movement. In this modern era of sports, the development of technological and technical clothing which can benefit runners especially the compression socks that is widely used by all runners in racing and training (Vercruyssen et al., 2014)

In recent years, distance running has become a popular physical and recreational activity (De Wit, De Clercq, \& Aerts, 2000). Thus, there was a demand to analyze different running kinematic parameters which are very important to set up new running strategies to achieve personal best or to win the race. Appropriate running mechanics involves synchronous movements of all the mechanisms in the kinetic chain. The running energy cost is not only determined by speed but also by running mechanics. However, previous research on compression socks was more focused on the physiological effects (Ali, Creasy, \& Edge, 2010; Kemmler et al., 2009) during the running performance. Today, limited research has been done on the use of compression socks on running kinematics. There is a need to investigate the potential usage of compression socks in improving the running kinematics among runners. Therefore, the purpose of this study was to determine the effects of wearing compression socks on running kinematics among runners. Besides, we sought to understand whether improved sensory afferent feedback from the foot, gained by wearing compression socks (Han, Waddington, Anson, \& Adams, 2013; Hasan et al., 2016; Hasan, Davids, Chow, \& Kerr, 2017), would enhance movement organization on the leg in performing graduated maximum velocity running. According to Han, Anson, Waddington and Adams (2014), sensory afferent feedback is also responsible for accuracy and involvement in the control of movement coordination in sports performance.

\subsection{Methodology}

\subsection{Participants}

16 recreational runners who participated in the study were aged between the range of $20-25(M=23.56, S D=1.41$ years old), with mixed experience in running from 5 to $70 \mathrm{~km}$ per week. All of the participants were required to be free from any injuries and any cardiovascularrelated diseases within the past six months before the testing session. Other than that, the participants had to be free from any alcohol or drug consumption 24-hours before the tests. All procedures in this study were approved by the Universiti Teknologi MARA ethical committee.

\subsection{Bruce Protocol}

All participants performed a total of 10 stages for a maximal treadmill exercise test with the first stage starting by walking at $2.74 \mathrm{~km} / \mathrm{h}$ and gradient set at 10\% (Jefry, Hasan, Azhan, Misnon, Azidin \& Ismail, 2019). The gradient and speed were increased every three minutes. Generally, the gradient increased by $2 \%$ at every level and the speed increased according to Bruce Protocol (Shah, 2013). If the participants achieve volitional fatigue or stop the exercise for any reason, the speed of the treadmill would reduce to $2.74 \mathrm{~km} / \mathrm{h}$ and the gradient was decreased to $0 \%$ indicating the end of the test. Participants end the test with another 5 minutes' walk for cooling down.

\subsection{Running Kinematics Analysis}

A high-speed camera (Olympus TG-5) was placed 4-meter away paralleled from the treadmill to obtain a sagittal view of the running movements (Jefry, Hasan, Azhan, Misnon, Azidin \& Ismail, 2019) and the video footage was set at 240 frames per second. Kinematics parameters measurements which included ground contact time (GCT), stride length (SL), swing time (ST), and heel strike (HS) were taken during the last 30 seconds of every 3 minutes' stages of the treadmill running test which is adopted from the study by (Stickford, Chapman, Johnston, \& Stager, 2015). Movements of the foot during running were analyzed using Kinovea 0.8.24, (France) movement analysis software.

\subsection{Procedures}

Two different periods of testing sessions were conducted for both conditions. The conditions were divided into Condition 1 (with compression socks) and Condition 2 (with smooth socks). Each participant underwent both conditions. The compression socks were constructed from nylon (85\%) and spandex (15\%), with a clinical compression level of 20-30 mmHg (Azhan, Hasan, Azidin, Misnon \& Ismail, 2020). The use of compression socks created higher compression of the soft tissues and receptors around the lower leg compared to smooth socks. The control (normal) socks were smooth socks that were of a similar thickness to the compression socks. In a single testing session, each group completed condition 1, and the other one completed condition 2. All participants underwent another running condition in the following sessions.

The maximal treadmill exercise test consists of 10 stages started with walking at $2.74 \mathrm{~km} / \mathrm{h}$ and a $10 \%$ gradient. It was increased in the following 3 minutes. The gradient increased by $2 \%$ on each of the stages, and the speed increased based on the Bruce Protocol that was set up by Robert Bruce in 1963. Participants who showed signs of volitional fatigue should stop. If participants need to stop during the test, they need to grab onto the treadmill handrails or by pressing the stop button located at the treadmill screen. Some of the reasons to stop the test include signs of poor perfusion or the respiratory exchange ratio was greater than or equal to 1.15. The treadmill speed was reduced to $2.74 \mathrm{~km} / \mathrm{h}$ and the gradient decreased to $0 \%$ as the test came to end.

\subsection{Data Analysis}

Descriptive statistics of an outcome measure would include means and standard deviations to characterize the participants. A correlational analysis (Pearson correlation) was used to determine whether there was any correlation between the kinematics parameters in the smooth and compression socks conditions. The strength of the relationship was considered of $r=0.0-0.1$ (very small), $r>0.1$ - 
$0.3, r=0.3-0.5$ (moderate); $r=0.5-0.7$ (strong); $r=0.7-0.9$ (very strong); $r=0.9-1$ (perfect). All significance levels were set at $\mathrm{p}<0.05$. All data were analyzed using the IBM Statistical Package for Social Sciences (SPSS V23.0, Chicago, USA).

\subsection{Findings}

Table 1 shows the means and standard deviations values (average of four stages) for the ground contact time, swing time, stride length, and heel strike under two socks conditions. Wearing compression socks during running improved all kinematic variables (Table 1) as compared to the smooth socks conditions. Table 2 shows the correlation coefficient values for smooth socks. Results revealed that there was a significant correlation ( $p=0.04, r=-0.518$ ) between GCT-SL when wearing normal socks during running (exhibiting a strong negative relationship). Other correlations in the smooth socks conditions showed very small negative relationships between the GCTST and ST-HS, moderate positive relationship between GCT-HS, and moderate negative relationship between SL-HS. For compression socks (Table 3), results revealed that significant correlations were found between ST-SL $(p=0.035, r=-0.529)$; and between ST-HS ( $p$ $=0.02, r=0.574$ ) with both correlations showing strong negative relationships. Other correlations in the compression socks conditions showed a very small negative relationship between the GCT-HS, moderate positive relationship between GCT-ST, moderate negative relationship between GCT-SL, and moderate negative relationship between HS-SL.

Table 1: Mean (SD) for ground contact time, swing time, stride length and heel strike under two socks conditions

\begin{tabular}{llllll}
\hline Conditions & Ground Contact Time $(\mathrm{s})$ & $\begin{array}{l}\text { Swing Time } \\
(\mathrm{s})\end{array}$ & $\begin{array}{l}\text { Stride Length } \\
(\mathrm{m})\end{array}$ & $\begin{array}{l}\text { Heel } \\
(\mathrm{s})\end{array}$ & Strike \\
\hline Smooth & & $0.62(0.05)$ & $1.50(0.04)$ & $0.18(0.01)$ \\
Compression & $0.55(0.03)$ & $0.58(0.02)$ & $1.56(0.03)$ & $0.17(0.10)$ \\
\hline
\end{tabular}

Table 2: Smooth socks correlation coefficient

\begin{tabular}{llllll}
\hline Movement Kinematics & & Ground Contact Time & Swing Time & Stride Length & Heel Strike \\
\hline Ground Contact Time & $r$ & - & -0.02 & -0.518 & 0.354 \\
\multirow{2}{*}{ Swing Time } & $p$ & - & 0.941 & $0.04^{*}$ & 0.178 \\
& $r$ & -0.02 & - & -0.29 & -0.24 \\
Stride Length & $p$ & 0.941 & - & 0.438 & 0.449 \\
& $r$ & -0.518 & -0.209 & - & -0.391 \\
Heel Strike & $p$ & $0.04^{*}$ & 0.438 & - & 0.134 \\
& $r$ & 0.354 & -0.204 & -0.391 & - \\
& $p$ & 0.178 & 0.449 & 0.134 & -
\end{tabular}

For this study, the use of smooth socks serves as the baseline data to be compared to the compression socks. Better kinematics parameters were recorded in the compression socks conditions. Results also revealed that using smooth socks during treadmill running only produced one significant correlation. When the runners use compression socks during treadmill running, this condition would produce more significant correlations as compared to the smooth socks. Besides, there were also more moderate relationships in the compression socks condition but the $p$ values just above the significance level. In the compression socks conditions, there were significant relationships between swing time and stride length/heel strike. Runners who run at high speed need to increase their stride length and heel strikes as this running kinematics will provide a better running economy. As found in similar studies by Kerhervé et al. (2017) reported that compression socks could be associated with higher aerial time and leg stiffness during running at a constant rate. Wearing compression socks would also affect the lower ground contact time with higher leg stiffness and higher vertical stiffness in the all-out running. It will affect the hopping movement that could lower the ground contact time (Kerhervé et al., 2017).

Table 3: Compression socks correlation coefficient

\begin{tabular}{lccccc}
\hline Movement Kinematics & & Ground Contact Time & Swing Time & Stride Length & Heel Strike \\
\hline Ground Contact Time & $r$ & - & 0.421 & -0.481 & -0.152 \\
Swing Time & $p$ & - & 0.104 & 0.059 & 0.575 \\
& $r$ & 0.421 & - & -0.529 & 0.574 \\
Stride Length & $p$ & 0.104 & - & $0.035^{*}$ & $0.02^{*}$ \\
& $r$ & -0.481 & -0.529 & - & -0.462 \\
Heel Strike & $p$ & 0.059 & $0.035^{*}$ & - & 0.072 \\
& $r$ & -0.152 & 0.574 & -0.462 & -
\end{tabular}




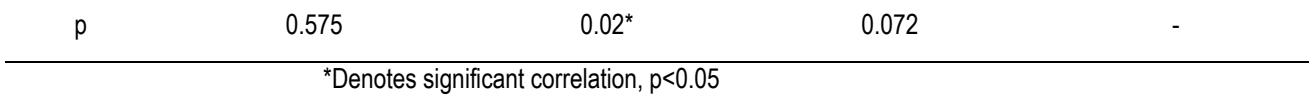

The explanatory rationale for these observations (i.e. significant relationship) among the participants in the compression socks condition is that these socks cause simple mechanical deformation and compressed the cutaneous sensory receptors (Orth et al., 2013; Sperlich, Born, Kaskinoro, Kalliokoski, \& Laaksonen, 2013). It also provides better lower limb positioning (Davids, Shuttleworth, Button, Renshaw, \& Glazier, 2004) due to increased haptic information from the foot. In addition, a study by Engel, Holmberg and Sperlich (2016) reported that compression socks exerted a small positive effect on biomechanical parameters which includes the swing time. This can be supported by the study from Bringard, Perrey and Belluye (2006) which reported that compression clothing improves neuromechanical parameters including recruitment of muscle fibers. From this outcome, the compression socks didn't only alter the running kinematics specifically for swing time. It also showed that there is a relationship between time spent in the air and also leg stiffness. In addition, there is a decrease in step frequency as their swing time increases. In supporting these results, Morin, Samozino, Zameziati and Belli (2005) concluded that increased step frequency results in decreased ground contact, vertical displacement and leg length variation while wearing the compression garment.

\subsection{Conclusion}

It is suggested that compression socks can be used by recreational runners to improve their running kinematics due to the significant relationships in the movement organizations found in this study. Besides, future research could compare the effects of wearing compression socks among novice and experienced runners. These distinct performers have their running movement patterns and it is interesting to find out whether enhanced somatosensory feedback by wearing compression socks could alter their specified running performance.

\section{Acknowledgements}

Special thanks to Lestari Grant UiTM (600-IRMI/Dana KCM 5/3/LESTARI (112/2017) for funding this research and grateful to those participants who contributed their time and helped to make this research possible.

\section{References}

Ali, A., Creasy, R. H., \& Edge, J. A. (2010). Physiological effects of wearing graduated compression stockings during running. European Journal of Applied Physiology, 109(6), 1017-1025

Areces, F., Salinero, J. J., Abian-Vicen, J., González-Millán, C., Ruiz-Vicente, D., Lara, B., Del Coso, J. (2015). The use of compression stockings during a marathon competition to reduce exercise-induced muscle damage: are they really useful? Journal of Orthopaedic and Sports Physical Therapy, 45(6), 462-470.

Azhan, M. A. N., Hasan, H., Misnon, M. I., Raja Azidin, R. M. F., \& Ismail, H. (2020). Effect of Compression Socks and Smooth Socks on VO2 max and Blood Lactate Concentration of Experienced and Novice Runners. Pertanika Journal of Social Sciences \& Humanities, 28(1).

Bringard, A., Perrey, S., \& Belluye, N. (2006). Aerobic energy cost and sensation responses during submaximal running exercise-positive effects of wearing compression tights. International Journal of Sports Medicine, 27(05), 373-378.

Davids, K., Shuttleworth, R., Button, C., Renshaw, I., \& Glazier, P. (2004). "Essential noise"-enhancing variability of informational constraints benefits movement control: a comment on Waddington and Adams (2003). British Journal of Sports Medicine, 38(5), 601-605.

De Wit, B., De Clercq, D., \& Aerts, P. (2000). Biomechanical analysis of the stance phase during barefoot and shod running. Journal of Biomechanics, 33(3), 269-278.

Han, J., Anson, J., Waddington, G., \& Adams, R. (2014). Sport attainment and proprioception. International Journal of Sports Science and Coaching, 9(1), 159-170.

Engel, F. A., Holmberg, H. C., \& Sperlich, B. (2016). Is there evidence that runners can benefit from wearing compression clothing? Sports Medicine, 46(12), $1939-1952$.

Han, J., Waddington, G., Anson, J., \& Adams, R. (2013). Level of competitive success achieved by elite athletes and multi-joint proprioceptive ability. Journal of Science and Medicine in Sport, 18, 77-81.

Hasan, H., Davids, K., Chow, J. Y., \& Kerr, G. (2016). Compression and texture in socks enhance football kicking performance. Human Movement Science, $48,102-111$. doi:http://dx.doi.org/10.1016/j.humov.2016.04.008

Hasan, H., Davids, K., Chow, J. Y., \& Kerr, G. (2017). Changes in organisation of instep kicking as a function of wearing compression and textured materials. European Journal of Sport Science, 17(3), 294-302.

Hooper, D. R., Dulkis, L. L., Secola, P. J., Holtzum, G., Harper, S. P., Kalkowski, R. J., Kraemer, W. J. (2015). The Roles of an Upper Body Compression Garment on Athletic Performances. Journal of Strength and Conditioning Research. DOI:10.1519/JSC.0000000000000909

Jefry, M. H., Hasan, H., Azhan, M. A. N., Misnon, M. I., Azidin, R. M. F. R., \& Ismail, H. (2019). The Effect of Compression Socks on Running Kinematics in Experience and Novice Runners. In the International Conference on Movement, Health and Exercise (pp. 333-340). Springer, Singapore. 
Kemmler, W., von Stengel, S., Köckritz, C., Mayhew, J., Wassermann, A., \& Zapf, J. (2009). Effect of compression stockings on running performance in men runners The Journal of Strength \& Conditioning Research, 23(1), 101-105.

Kerhervé, H. A., Samozino, P., Descombe, F., Pinay, M., Millet, G. Y., Pasqualini, M., \& Rupp, T. (2017). Calf compression sleeves change biomechanics but not performance and physiological responses in trail running. Frontiers in Physiology, 8, 247.

Kraemer, W. J., French, D. N., \& Spiering, B. A. (2004). Compression in the treatment of acute muscle injuries in sport. International Sport and Medicine Journal, 5(3) 200-208.

Morin, J. B., Dalleau, G., Kyröläinen, H., Jeannin, T., \& Belli, A. (2005). A simple method for measuring stiffness during running. Journal of Applied Biomechanics, 21(2), $167-180$.

Orth, D., Davids, K., Wheat, J., Seifert, L., Liukkonen, J., Jaakkola, T., Kerr, G. (2013). The role of textured material in supporting perceptual-motor functions. PLoS ONE, 8(4), 1-14. DOI:10.1371/journal.pone.0060349

Shah, B. N. (2013). On the 50th anniversary of the first description of a multistage exercise treadmill test: re-visiting the birth of the 'Bruce protocol'. In: BMJ Publishing Group Ltd and British Cardiovascular Society.

Sperlich, B., Born, D.-P., Kaskinoro, K., Kalliokoski, K. K., \& Laaksonen, M. S. (2013). Squeezing the muscle: compression clothing and muscle metabolism during recovery from high-intensity exercise. PLOS ONE, 8(4), e60923.

Stickford, A. S., Chapman, R. F., Johnston, J. D., \& Stager, J. M. (2015). Lower-leg compression, running mechanics, and economy in trained distance runners International Journal of Sports Physiology and Performance, 10(1), 76-83.

Vercruyssen, F., Easthope, C., Bernard, T., Hausswirth, C., Bieuzen, F., Gruet, M., \& Brisswalter, J. (2014). The influence of wearing compression stockings on performance indicators and physiological responses following a prolonged trail running exercise. European Journal of Sport Science, 14(2), 144-150.

Woo, M. T., Davids, K., Liukkonen, J., Jaakkola, T., \& Chow, J. Y. (2014). Effects of Textured Compression Socks on Postural Control in Physically Active Elderly Individuals. Procedia Engineering, 72, 162-167. DOl:10.1016/j.proeng.2014.06.028 